\title{
Proteomics reveals urine apolipoprotein A-I as a potential biomarker of acute kidney injury following percutaneous coronary intervention in elderly patients
}

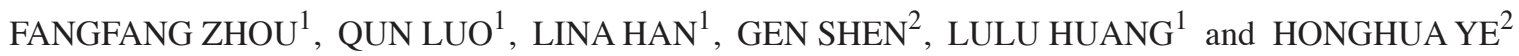 \\ Departments of ${ }^{1}$ Nephrology and ${ }^{2}$ Cardiology, HwaMei Hospital, \\ University of Chinese Academy of Sciences, Ningbo, Zhejiang 315010, P.R. China
}

Received December 18, 2019; Accepted April 14, 2021

DOI: $10.3892 /$ etm.2021.10177

\begin{abstract}
The aim of the present study was to investigate how changes in the lipid composition are involved in early stages of acute kidney injury (AKI) following percutaneous coronary intervention (PCI-AKI) in elderly patients. A prospective nested case-control study was performed. Alterations in the urine protein accumulation were investigated in patients with and without PCI-AKI using isobaric tags for relative and absolute quantitation (iTRAQ). In addition, differentially expressed proteins (DEPs) related to lipids were confirmed using parallel reaction monitoring (PRM)-based targeted proteomics. From the cohort of elderly patients ( $>60$ years of age), 14 (12.28\%) developed AKI within $48 \mathrm{~h}$ after PCI. No significant differences were detected between the AKI and control (CON) groups for serum creatinine at $24 \mathrm{~h}$ following treatment $(\mathrm{P}=0.27)$. Among the DEPs that overlapped in both the AKI-24 h/AKI-Pre (AKI group at $24 \mathrm{~h}$ post-PCI vs. pre-PCI) and $\mathrm{AKI}-24 \mathrm{~h} / \mathrm{CON}-24 \mathrm{~h}$ groups (AKI group vs. CON group at $24 \mathrm{~h}$ post-PCI), only apolipoprotein A-I (apoA-I) was related to lipids, which displayed a significant upregulation in expression levels. The protein expression levels of apoA-I displayed a 5.98-fold increase at $24 \mathrm{~h}$ after PCI from the baseline and a 2.09-fold increase compared with the control group as determined using PRM, which exhibited a similar trend to the iTRAQ results. Using protein-protein interaction analyses, apoA-I was determined to be functionally linked to the complement and coagulation cascades, the renin-angiotensin system and the hypoxia-inducible factor-1 signaling pathway. Using the pathway analysis tool from the Kyoto Encyclopedia of Genes and Genomes, several pathways were identified to be associated with apoA-I, including fat digestion and absorption,
\end{abstract}

Correspondence to: Dr Qun Luo, Department of Nephrology, HwaMei Hospital, University of Chinese Academy of Sciences, 41 Xibei Street, Ningbo, Zhejiang 315010, P.R. China

E-mail: nbeyluoqun@126.com

Key words: percutaneous coronary intervention, acute kidney injury, proteomics, apolipoprotein A-I, biomarker vitamin digestion and absorption, as well as the peroxisome proliferator activated receptor signaling pathway. In conclusion, apoA-I may be a promising biomarker for the early diagnosis of PCI-AKI in elderly patients. The role of apoA-I in the pathobiology of PCI-AKI requires further exploration.

\section{Introduction}

Percutaneous coronary intervention (PCI) is being performed on an increasing number of patients. Despite recent advances with radiocontrast agents, acute kidney injury (AKI) is still a major complication of PCI, representing the third most common cause of hospital-acquired acute kidney injury (AKI) $(1,2)$. The incidence of PCI-AKI ranges from $2 \%$ in the healthy population to as high as $30 \%$ in high-risk patients with pre-existing conditions, including impaired renal function, diabetes and older age ( $>60-75$ years). PCI-AKI is associated with a short- and long-term increase in morbidity, such as the development of end-stage renal disease, and mortality rates $(3,4)$.

Progress in improving the prognosis of PCI-AKI has been limited. This is in part due to an incomplete understanding of the pathophysiology of the disease process and the scarcity of early biomarkers for the diagnosis of PCI-AKI (5). Recently, a considerable number of novel biomarkers for the early diagnosis of PCI-AKI have been developed, such as neutrophil gelatinase-associated lipocalin (NGAL), interleukin-18 (IL-18) and kidney injury molecule-1 (KIM-1) (6). In a previous study, a rise in urine NGAL after $6 \mathrm{~h}$ and an increase in urine KIM-1 and IL-18 levels after $24 \mathrm{~h}$ post-PCI was determined (7). However, to date, there is no single proven ideal biomarker. Therefore, it is important to screen for ideal biomarkers with high sensitivity and specificity. Fortunately, advances in clinical proteomics have markedly accelerated the rate of discovery of candidate biomarkers and have provided novel insight into the pathways and biological processes that may hold promise as potential therapeutic targets for the treatment of PCI-AKI $(8,9)$. Isobaric tags for relative and absolute quantitation (iTRAQ) coupled with liquid chromatography tandem mass spectrometry (LC-MS/MS) have been recently applied as powerful proteomics tools (10). 
Previous studies have indicated that AKI may cause lipid dysfunction and abnormal lipid accumulation within the kidney, which may be either protective or toxic to the kidney depending on the nature of the specific lipid present and the time course of the injury (11). Studies have also demonstrated that lower levels of high-density lipoprotein cholesterol (HDL-C) are associated with an increased risk of AKI and long-term mortality in patients undergoing PCI or revascularization for chronic limb ischemia $(12,13)$. Despite previous studies demonstrating a benefit of pre-procedural statin for reducing the risk of PCI-AKI, the role of lipids in the development of PCI-AKI has remained to be fully elucidated. To explore the changes in lipid composition that are involved in the early stage of PCI-AKI, which may be pathophysiologically relevant and/or have the potential to serve as an early biomarker, the accumulation of urine protein in patients with and without PCI-AKI were investigated in the present study using the iTRAQ LC-MS/MS approach. In addition, differentially expressed proteins (DEPs) related to the expression of various lipids were further confirmed using parallel reaction monitoring (PRM), which has been widely used to quantify and detect target proteins (14).

\section{Patients and methods}

Study population. The present study was a single-center, prospective nested, case-control study. A total of 114 elderly patients undergoing elective PCI at HwaMei Hospital, University of Chinese Academy of Sciences (Ningbo, China) between July 2015 and June 2016 were eligible for enrollment. The exclusion criteria for the present study were as follows: i) Age $<60$ years; ii) pre-existing estimated glomerular filtration rate $(\mathrm{eGFR})<30 \mathrm{ml} / \mathrm{min} / 1.73 \mathrm{~m}^{2}$ or dialysis; iii) established AKI; iv) liver failure; v) tumors, vi) cardiogenic or septic shock; vii) emergency PCI; viii) exposure to contrast medium $48 \mathrm{~h}$ prior to study; and ix) use of nephrotoxic drugs at least 1 week prior to or during the study period. Each enrolled patient was given the non-ionic, iso-osmolar contrast medium Iodixanol (GE Healthcare) and intravenous hydration after PCI, without any specific protocols and medications for AKI prevention. The present study was approved by the ethics committee of HwaMei Hospital, University of Chinese Academy of Sciences (Ningbo, China). Written informed consent was obtained from each of the patients involved in the study.

Criteria for AKI were according to the Kidney Disease, Improving Global Outcomes guidelines from 2012 (15) and were applied for the diagnosis of PCI-AKI, defined as an absolute rise in serum creatinine (Scr) $\geq 0.3 \mathrm{mg} / \mathrm{dl}(26.5 \mu \mathrm{mol} / \mathrm{l})$ within 48 h or a $\geq 50 \%$ increase from the baseline value within 7 days after the procedure. The urine criteria for AKI were not used in the present study as urine output may be affected by previous medical therapies.

Cases and controls. Of the 114 subjects, 14 elderly patients with AKI (12.28\%) were assigned to the AKI-group. A total of 14 patients [the control (CON) group], 1:1 matched by age and sex, were selected from the remaining 100 patients who did not develop AKI (the non-AKI group) in the cohort.
Urine sample collection and storage. From the total cohort $(\mathrm{n}=114)$, serial urine samples were collected prospectively pre-PCI and $24 \mathrm{~h}$ post-PCI. Samples were centrifuged at $3,500 \mathrm{x} \mathrm{g}$ for $15 \mathrm{~min}$ at $4^{\circ} \mathrm{C}$. The supernatants were stored at $-80^{\circ} \mathrm{C}$ until use. Finally, 56 urine samples from the AKI group $(n=14)$ and CON group $(n=14)$ were used (28 patients at 2 time-points). The samples were divided into a discovery set, comprising 24 urine samples from 6 AKI- and 6 CON-group patients at 2 time-points; and a validation set of 32 urine samples from $8 \mathrm{AKI}$ - and $8 \mathrm{CON}$-group patients at 2 time-points.

Clinical and laboratory data. Basic data were collected, including demographics, medical history, use of medications, number of diseased vessels, the contrast volume used and left ventricular ejection fraction (LVEF) as measured by echocardiography, as well as laboratory findings including Scr levels prior to, as well as 24 and $48 \mathrm{~h}$ after PCI, as measured using an automated biochemistry analyzer (DXC800; Beckmann Coulter, Inc.) at the hospital's clinical laboratory. The eGFR was calculated using the Chronic Kidney Disease (CKD) Epidemiology Collaboration formula (16).

Sample preparation. For the discovery phase, 24 urine samples from the discovery set were used. Pooled urine samples $(6-7 \mathrm{ml})$ were centrifuged at $2,000 \mathrm{x} \mathrm{g}$ for $10 \mathrm{~min}$ at $4^{\circ} \mathrm{C}$ to remove cell debris. The supernatants were concentrated in Amicon Ultra-15 Centrifugal Filters (EMD Millipore) at $4,000 \mathrm{x} \mathrm{g}$ for $60 \mathrm{~min}$ at $4^{\circ} \mathrm{C}$. The concentrated solutes were recovered from the filter device. Proteins were precipitated by adding five volumes of pre-chilled $\left(-20^{\circ} \mathrm{C}\right)$ acetone and freezing at $-20^{\circ} \mathrm{C}$ overnight. Samples were centrifuged at $12,000 \mathrm{x} \mathrm{g}$ for $15 \mathrm{~min}$ at $4^{\circ} \mathrm{C}$. Acetone was carefully removed and $1 \mathrm{ml}$ of pre-chilled acetone was added. The samples were vortexed for $15 \mathrm{~min}$ twice to wash the pellets. The samples were centrifuged at $12,000 \mathrm{x}$ for $15 \mathrm{~min}$ at $4^{\circ} \mathrm{C}$ and subsequently, the acetone was decanted. The pellets were then left to dry at room temperature.

Protein digestion. Protein was dissolved in 100 buffer (7 M urea, $2 \mathrm{M}$ thiourea, $120 \mathrm{mM}$ dithiothreitol and $40 \mathrm{mM}$ Tris) and incubated at $37^{\circ} \mathrm{C}$ for $15 \mathrm{~min}$. Protein concentrations were determined using Bradford protein assays. A total of $60 \mu \mathrm{g}$ protein per condition was added into a new tube and the final volume was adjusted to $100 \mu \mathrm{l}$ with $100 \mathrm{mM}$ triethylammonium bicarbonate (TEAB). A total of $1 \mu 11 \mathrm{M}$ Tris (2-carboxyethyl) phosphine was added to each sample, followed by incubation at $55^{\circ} \mathrm{C}$ for $30 \mathrm{~min}$. Subsequently, $2 \mu 11 \mathrm{M}$ iodoacetamide was added to each sample, followed by incubation in the dark for $30 \mathrm{~min}$ at room temperature. Proteins were precipitated by adding five volumes of pre-chilled $\left(-20^{\circ} \mathrm{C}\right)$ acetone and freezing at $-20^{\circ} \mathrm{C}$ overnight. Samples were centrifuged at $12,000 \mathrm{x}$ g for $15 \mathrm{~min}$ at $4^{\circ} \mathrm{C}$. The acetone was carefully removed and $1 \mathrm{ml}$ pre-chilled $50 \%$ ethanol $/ 50 \%$ acetone was added, followed by vortexing twice for $10 \mathrm{~min}$ to wash the pellets. The samples were centrifuged at $12,000 \times \mathrm{g}$ for $10 \mathrm{~min}$ at $4^{\circ} \mathrm{C}$ and the ethanol/acetone was decanted. The pellets were then allowed to dry at room temperature. The precipitated protein pellets were resuspended in $100 \mu \mathrm{l}$ of $50 \mathrm{mM}$ TEAB. Proteins were digested using sequence-grade modified trypsin $(1: 50 \mathrm{w} / \mathrm{w}$; 
Promega Corp.) at $37^{\circ} \mathrm{C}$ overnight. The peptide mixture was lyophilized using a vacuum concentrator and then dissolved with $0.5 \mathrm{M}$ TEAB for subsequent iTRAQ labeling.

iTRAQ labeling. The resultant peptide mixture was labeled with iTRAQ 8-Plex reagent (SCIEX) following the manufacturer's protocol. The labeled peptide samples were then pooled and lyophilized in a vacuum concentrator.

High-pH reverse-phase separation. The peptide mixture was re-dissolved in buffer A, consisting of $20 \mathrm{mM}$ ammonium formate in water ( $\mathrm{pH} 10.0$ ), adjusted using ammonium hydroxide, and then fractionated by high-pH separation using an Ultimate 3000 system (Thermo Fisher Scientific, Inc.) connected to a reverse-phase column (XBridge C18 column; 4.6x250 mm, $5 \mu \mathrm{m}$; Waters Corp.). High pH separation was performed using a linear gradient. Starting from 5-45\% solution B in $40 \mathrm{~min}$ (solution B consisted of $20 \mathrm{mM}$ ammonium formate in $80 \%$ acetonitrile $(\mathrm{ACN})(\mathrm{pH} 10.0)$, adjusted with ammonium hydroxide. The column was re-equilibrated at the initial conditions for $15 \mathrm{~min}$. The column flow rate was maintained at $1 \mathrm{ml} / \mathrm{min}$ and the column temperature was maintained at $30^{\circ} \mathrm{C}$. In total, 12 fractions were collected. Each fraction was dried in a vacuum concentrator for the next step.

Low-pH nano-HPLC-MS/MS analysis. The fractions were resuspended in $30 \mu \mathrm{l}$ solvent $\mathrm{C}$, which consisted of water with $0.1 \%$ formic acid, separated using nanoLC and analyzed by on-line electrospray tandem mass spectrometry. The experiments were performed on an Easy-nLC 1000 system (Thermo Fisher Scientific, Inc.) connected to an Orbitrap Fusion Tribrid mass spectrometer (Thermo Fisher Scientific, Inc.) equipped with an online nano-electrospray ion source. A total of $10 \mu \mathrm{l}$ peptide sample was loaded onto the trap column (Acclaim PepMap C18; $100 \mu \mathrm{m}$ x $2 \mathrm{~cm}$; Thermo Fisher Scientific, Inc.) with a flow of $10 \mu \mathrm{l} / \mathrm{min}$ for $3 \mathrm{~min}$ and subsequently separated on the analytical column (Acclaim PepMap C18; $75 \mu \mathrm{m} \times 15 \mathrm{~cm}$; Thermo Fisher Scientific, Inc.) with a linear gradient, from 3-32\% phase B (phase B consisted of ACN with $0.1 \%$ formic acid), and phase A was water (with $0.1 \%$ formic acid) in $120 \mathrm{~min}$. The column was re-equilibrated to the initial conditions for $10 \mathrm{~min}$. The column flow rate was maintained at $300 \mathrm{nl} / \mathrm{min}$. The electrospray voltage of $2 \mathrm{kV}$ vs. the inlet of the mass spectrometer was used. The mass spectrometer was run in the data-dependent acquisition mode and automatically switched to the MS and MS/MS mode. The MS1 mass resolution was set to $120 \mathrm{~K}$ with a mass-to-charge ratio $(\mathrm{m} / \mathrm{z})$ of $350-1,550$ and the MS/MS resolution was set to $30 \mathrm{~K}$ under the high energy collision dissociation (HCD) mode. The dynamic exclusion time was set as $30 \mathrm{sec}$.

Data analysis. Tandem mass spectra were processed using PEAKS Studio version 8.5 (Bioinformatics Solutions, Inc.). PEAKS DB was set up to search the UniProt database (https://www.uniprot.org/proteomes/UP000005640; 70,956 entries) assuming the digestion enzyme trypsin. The PEAKS DB search was performed with a fragment ion mass tolerance of $0.05 \mathrm{Da}$ and a parent ion tolerance of 7.0 ppm. Carbamidomethylation, iTRAQ 8plex (K, N-term) was specified as a fixed modification. Oxidation (M), deamidation (NQ), acetylation (protein N-term), acetylation $(\mathrm{K})$, acetylation $(\mathrm{N}$-term), amidation, $\beta$-methylthiolation, biotinylation and 304 more modifications were specified as variable modifications. Peptides were filtered with a peptide hit threshold $(-10 \log P)$ of 30.0. PEAKS Q was used for peptide and protein abundance calculations. Normalization was performed on the average levels of all peptides. Medians were used for averaging. DEPs were filtered if their fold change was $>1.2$, with $\mathrm{P}<0.05$.

Biological information function analysis of the DEPs. Blast2GO version 4 (www.blast2go.com) was used for functional annotations. The whole protein sequence database (The Uniprot Human database used for DB search) was analyzed using BlastP (https://blast.ncbi.nlm.nih.gov/Blast. cgi?PROGRAM=blastp\&PAGE_TYPE=BlastSearch\&LINK_ LOC=blasthome), then all human sequences in the Uniprot database were mapped for annotation background and DEPs were used for Fisher's exact test with the Gene Ontology (GO) database (www.geneontology.org). Statistically altered functions of DEPs were calculated using Fisher's exact tests in BLAST2GO (https://www.blast2go.com). The intersections of the DEPs between AKI-24 h/AKI-Pre and AKI-24 h/CON-24 h were presented using a Venn diagram. The protein-protein interaction network was generated using the Search Tool for the Retrieval of Interacting Genes and proteins (STRING) database (http.//string-db.org/) and Cytoscape software (http.//www. cytoscape.org/; version 3.2.1). Kyoto Encyclopedia of Genes and Genomes (KEGG) pathway mapping was performed using the KEGG database (http./www.kegg.jp/kegg/pathway.html).

PRM MS acquisition. Targeted quantification and verification was performed on 32 urine samples from the discovery set, across 6 different operational conditions. The DEPs that were related to the lipid composition were selected, with 3-7 peptides per protein and the peptide information was from a previous data-dependent acquisition and Selected Reaction Monitoring Atlas database (http://www.srmatlas.org/). Only peptides meeting the following conditions were selected for target proteomics: i) Fully digested with trypsin (0 missed cleavages); and ii) $\geq 7$ amino acids. PRM analysis was performed using the Orbitrap Fusion ${ }^{\mathrm{TM}}$ Tribrid $^{\mathrm{TM}}$ (Thermo Fisher Scientific, Inc.). Approximately $0.5 \mu \mathrm{g}$ of urine protein was separated and eluted with a linear 120-min gradient of 2-35\% solvent $\mathrm{B}(0.1 \% \mathrm{v} / \mathrm{v}$ formic acid in $\mathrm{CAN})$, and solvent $\mathrm{A}$ was water (with $0.1 \%$ formic acid) at a flow rate of $300 \mathrm{nl} / \mathrm{min}$. After each gradient, the column was flushed with $80 \%$ solvent B for $10 \mathrm{~min}$ and equilibrated with $2 \%$ solvent B for another $20 \mathrm{~min}$. The MS acquisition mode was a combination of two scan events, namely a full scan and a time-scheduled scan. The full scan was taken with a resolution of 60,000 at $\mathrm{m} / \mathrm{z} 200$, with a scan mass range of $\mathrm{m} / \mathrm{z} 270-1,470$. An automatic gain control (AGC) target of $2 \times 10^{5}$ was used and the maximum injection fill time was $100 \mathrm{msec}$. The non-scheduled scan was employed with a resolution of 15,000 at m/z 200. A target AGC of $5 \times 10^{4}$ was used and the maximum injection fill time was $60 \mathrm{msec}$. The precursor ion of each target peptide was isolated with a 2-Da window. Precursor ions were fragmented with an HCD normalized collision energy of $35 \%$. 
PRM MS data analysis. MS raw files (raw) from parallel reaction monitoring acquisition were first analyzed using the Trans-Proteomic Pipeline software suite version 4.7.0 (TPP; Institute for Systems Biology). Raw files were first converted to mzXML and the MS/MS spectra acquired were searched using Comet version 2.0 (http://comet-ms.sourceforge.net/) against the Swissprot human canonical database (https://www.uniprot. org/uniprot/?query=proteome:UP000005640\%20reviewed:yes; 20,192 entries) with reversed sequences. Common contaminants were included. Searching parameters were set as follows: i) Precursor ion mass tolerance, $\pm 10 \mathrm{ppm}$; ii) fragment ion mass tolerance, $0.02 \mathrm{Da}$; iii) semi-tryptic termini and two missing cleavages were allowed; iv) fixed modification, carbamidomethylation; and v) dynamic modification, oxidation. Search results were further processed using PeptideProphet embedded in TPP. Peptide probabilities were calculated and the false discovery rate for each peptide was set to 0.01 . The generated interact.pep.xml files were imported into Skyline version 3.1.0 to create a library and the cut-off score was set as 0.90 . The list of target precursors was then uploaded to Skyline and the 6 most intense product ions matching the library were selected as transitions. Peak picking was manually checked and corrected according to the transitions, retention time, mass accuracy and MS/MS spectra. The relative protein abundance was defined as the average intensity of peptides.

Statistical analysis. Continuous variables were tested for normality of distribution using the Shapiro-Wilk W and Kolmogorov-Smirnov tests and are expressed as the mean \pm standard deviation or as medians with interquartile ranges (25-75th percentile). When comparing two groups, a two-samples t-test, or Mann-Whitney U test were used to compare continuous variables. Categorical variables are expressed as $\mathrm{n}(\%)$ and comparisons between groups were performed using the $\chi^{2}$ test or Fisher's exact test. ANOVA followed by the least-significant difference test was used to evaluate significant differences among different time-points. $\mathrm{P}<0.05$ was considered to indicate a statistically significant difference. Statistical analyses were performed using SPSS for Windows version 22.0 (IBM Corp.).

\section{Results}

Patient demographics. Among the patients of the present study (mean age, 74.57 \pm 6.13 years, $53.57 \%$ males), $13(92.86 \%)$ developed AKI within $48 \mathrm{~h}$ after PCI. One patient $(7.14 \%)$ developed AKI within 7 days after PCI. None of the patients in the cohort with or without AKI required renal replacement therapy or died during their hospitalization. Table I displays the demographic and clinical characteristics of the AKI group, CON group (selected from the non-AKI group) and the non-AKI group. There were no significant differences between the AKI group and CON group with respect to sex, age, body mass index, smoking status, hypertension, diabetes, chronic kidney disease (CKD), hemoglobin, albumin, baseline Scr, baseline eGFR, brain natriuretic peptide, LVEF, use of medications (aspirin, angiotensin-converting enzyme inhibitors/angiotensin II receptor blockers, calcium channel blockers, $\beta$-blockers, diuretics and statins), dosage of statins, number of diseased vessels and contrast volume. Regarding the lipid profile, only high-density lipoprotein (HDL)-cholesterol $(\mathrm{P}=0.023)$ and apolipoprotein (apo)A-I $(\mathrm{P}=0.047)$ levels were lower in the AKI group. There were no significant differences between the CON-group and non-AKI group regarding the baseline levels of any other parameter, including the lipid profile.

Regarding the Scr levels at $24 \mathrm{~h}$ post-contrast, no significant differences were detected in the AKI-24 h vs. AKI-Pre $(\mathrm{P}=0.243)$ and $\mathrm{AKI}-24 \mathrm{~h}$ vs. $\mathrm{CON}-24 \mathrm{~h}$ groups $(\mathrm{P}=0.270)$. However, there was a significant rise at $48 \mathrm{~h}$ post-contrast, with AKI-48 h vs. AKI-Pre (P=0.011) and AKI-48 h vs. CON-48 h $(\mathrm{P}=0.006)$. The Scr levels did not significantly change at any time-point in the control group (CON-24 h vs. CON-Pre, $\mathrm{P}=0.723$; $\mathrm{CON}-48$ h vs. CON-Pre, $\mathrm{P}=0.664$; Table I).

DEPs obtained by iTRAQ-2D-LC-MS/MS. According to the criteria used in the present study for judging DEPs (fold change $>1.2$ or $<0.83$ and $\mathrm{P}<0.05$ ), 106 and 77 upregulated proteins were identified for the AKI-24 h/AKI-Pre and AKI-24 h/CON-24 h comparisons, respectively. In addition, 61 and 114 downregulated proteins were identified (Fig. 1A). All DEPs in the AKI-24 h/AKI-Pre and AKI-24 h/CON-24 h comparisons are presented in Table SI.

As illustrated in the Venn diagram in Fig. 1B, 94 proteins were overlapped in both the AKI-24 h/AKI-Pre and AKI-24 h/CON-24 h comparisons. Among them, only apoA-I [Accession no. splP02647IAPOA1_HUMAN; Peptides (95\%), 21; Table SI] was related to lipids, displaying significantly upregulated levels in both the AKI-24 h/AKI-Pre and AKI-24 h/CON-24 h comparisons, while no significant differences were observed for both the CON-24 h/CON-Pre and AKI-Pre/CON-Pre comparisons (Table II). It is worth mentioning that the fold changes observed for apoA-I were striking before the Scr levels were raised, because as mentioned above, no significant differences were detected in the comparisons between the AKI-24 h/AKI-Pre $(\mathrm{P}=0.243)$ and AKI-24 h/CON-24 h $(\mathrm{P}=0.270)$ in $\mathrm{Scr}$ at $24 \mathrm{~h}$ post-contrast.

Bioinformatics analyses of urinary proteome. The 20 most significantly enriched GO terms and their percentages in the enrichment results of 3 of the basic categories are presented in Fig. 2A and B. All statistically significant GO terms in the categories biological process, cellular component and molecular function are listed in Tables SII and SIII. The most relevant terms were 'extracellular region' in the category cellular component, 'response to stress' in biological process and 'binding' in molecular function. According to the KEGG pathway analysis, 'lysosome', 'complement' and 'coagulation cascades' were the most relevant in the AKI-24 h/AKI-Pre and AKI-24 h/CON-24 h comparisons (Fig. 3A and B). All statistically significant KEGG pathways are listed in Tables SIV and SV.

Bioinformatics analyses of apoA-I. The interaction network was illustrated using Cytoscape software. Differentially expressed nodes identified from the iTRAQ analysis between the AKI-24 h and AKI-Pre samples were used as input. A force-directed layout that was restricted to the selected node of apoA-I and its first neighbors was produced, based on the interactions between apoA-I and proteins provided 
Table I. Baseline clinical characteristics of patients undergoing elective percutaneous coronary intervention in the AKI ( $\mathrm{n}=14)$, control $(\mathrm{n}=14)$ and non-AKI $(\mathrm{n}=100)$ groups.

\begin{tabular}{|c|c|c|c|c|c|}
\hline Item & AKI group & Control group & Non-AKI group & P-value & P-value ${ }^{b}$ \\
\hline Male sex & $8(53.3)$ & $7(50.0)$ & $67(67.0)$ & 0.715 & 0.348 \\
\hline Age, years & $74.21 \pm 7.99$ & $74.93 \pm 5.96$ & $71.45 \pm 10.01$ & 0.797 & 0.225 \\
\hline $\mathrm{BMI}, \mathrm{kg} / \mathrm{m}^{2}$ & $24.18 \pm 2.94$ & $22.39 \pm 3.17$ & $24.01 \pm 3.53$ & 0.142 & 0.118 \\
\hline Smoking & $2(14.3)$ & $2(14.3)$ & $17(16.0)$ & 1.000 & 0.445 \\
\hline Hypertension & $12(85.7)$ & $9(64.3)$ & $73(73.0)$ & 0.190 & 0.774 \\
\hline Diabetes mellitus & $4(28.6)$ & $5(35.7)$ & $30(30.0)$ & 0.686 & 0.535 \\
\hline CKD & $4(28.6)$ & $3(21.4)$ & $17(17.0)$ & 0.663 & 0.589 \\
\hline Hemoglobin, g/l & $120.21 \pm 13.70$ & $115.62 \pm 12.03$ & $122.94 \pm 16.50$ & 0.365 & 0.211 \\
\hline Albumin, g/l & $36.91 \pm 4.62$ & $39.45 \pm 4.43$ & $39.45 \pm 4.58$ & 0.158 & 0.999 \\
\hline Baseline eGFR, $\mathrm{ml} / \mathrm{min} / 1.73 \mathrm{~m}^{2}$ & $72.13 \pm 22.42$ & $68.66 \pm 19.79$ & $77.34 \pm 18.86$ & 0.674 & 0.075 \\
\hline \multicolumn{6}{|l|}{$\mathrm{Scr}, \mu \mathrm{mol} / 1$} \\
\hline Baseline & $85.42 \pm 29.30$ & $90.40 \pm 29.42$ & $82.30 \pm 25.86$ & 0.664 & 0.298 \\
\hline Post-24 h & $102.41 \pm 44.37$ & $86.83 \pm 25.02^{c}$ & $82.54 \pm 25.35$ & 0.270 & 0.569 \\
\hline Post-48 h & $123.71 \pm 38.66$ & $87.30 \pm 22.05^{\mathrm{d}}$ & $83.07 \pm 23.58$ & 0.006 & 0.545 \\
\hline $\mathrm{BNP}, \mathrm{pg} / \mathrm{ml}$ & $6258(394,8248)$ & $897(394,1968)$ & $544(278,1032)$ & 0.123 & 0.180 \\
\hline LVEF, \% & $60.00 \pm 7.90$ & $60.38 \pm 7.25$ & $63.34 \pm 8.16$ & 0.896 & 0.218 \\
\hline \multicolumn{6}{|l|}{ Lipid profile } \\
\hline $\mathrm{TC}, \mathrm{mmol} / \mathrm{l}$ & $3.76 \pm 0.65$ & $3.55 \pm 1.43$ & $3.86 \pm 1.07$ & 0.614 & 0.284 \\
\hline $\mathrm{TG}, \mathrm{mmol} / \mathrm{l}$ & $1.50 \pm 0.89$ & $1.16 \pm 0.40$ & $1.51 \pm 0.11$ & 0.205 & 0.129 \\
\hline HDL-C, mmol/1 & $0.99 \pm 0.27$ & $1.32 \pm 0.43$ & $1.21 \pm 0.28$ & 0.023 & 0.198 \\
\hline LDL-C, mmol/1 & $2.07 \pm 0.66$ & $2.01 \pm 0.80$ & $2.06 \pm 0.89$ & 0.808 & 0.330 \\
\hline ApoA-I, g/l & $1.09 \pm 0.23$ & $1.27 \pm 0.21$ & $1.22 \pm 0.18$ & 0.047 & 0.542 \\
\hline ApoB, g/l & $0.83 \pm 0.22$ & $0.69 \pm 0.19$ & $0.83 \pm 0.28$ & 0.113 & 0.078 \\
\hline ApoE, mg/dl & $4.37 \pm 1.84$ & $4.67 \pm 1.18$ & $4.71 \pm 1.70$ & 0.624 & 0.760 \\
\hline Lipoprotein (a), mg/l & $202.23 \pm 157.85$ & $207.63 \pm 167.97$ & $233.75 \pm 224.61$ & 0.932 & 0.489 \\
\hline \multicolumn{6}{|l|}{ Medication } \\
\hline Aspirin & $14(100)$ & $14(100)$ & $100(100)$ & 1.000 & 1.000 \\
\hline ACEI/ARB & $9(64.3)$ & $8(57.1)$ & $67(67.0)$ & 0.699 & 0.776 \\
\hline $\mathrm{CCB}$ & $4(28.6)$ & $3(21.4)$ & $17(17.0)$ & 0.663 & 0.589 \\
\hline$\beta$-blocker & $12(85.7)$ & $10(71.4)$ & $71(71.0)$ & 0.357 & 0.942 \\
\hline Diuretics & $9(64.3)$ & $7(50.0)$ & $37(37.0)$ & 0.445 & 0.241 \\
\hline Statins & $14(100)$ & $14(100)$ & $100(100)$ & 1.000 & 1.000 \\
\hline \multicolumn{6}{|l|}{ Statin dosage, mg } \\
\hline Atorvastatin & $24.00 \pm 8.43$ & $22.00 \pm 6.32$ & $24.00 \pm 8.58$ & 0.556 & 0.441 \\
\hline Rosuvastatin & $12.50 \pm 5.00$ & $13.33 \pm 5.77$ & $12.52 \pm 6.11$ & 0.846 & 0.849 \\
\hline Number of diseased vessels & $2.21 \pm 0.80$ & $2.00 \pm 0.91$ & $2.03 \pm 0.83$ & 0.522 & 0.904 \\
\hline Contrast volume, $\mathrm{ml}$ & $182.86 \pm 46.81$ & $180.00 \pm 39.79$ & $168.30 \pm 32.23$ & 0.866 & 0.233 \\
\hline
\end{tabular}

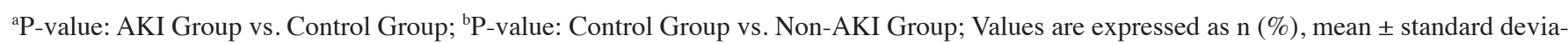
tion or the median $(25,75$ th percentile). AKI, acute kidney injury; BMI, body mass index; CKD, chronic kidney disease; Scr, serum creatinine; eGFR, estimated glomerular filtration rate; BNP, brain natriuretic peptide; LVEF, left ventricular ejection fraction; TC, total cholesterol; TG, total triglycerides; HDL-C, high-density lipoprotein cholesterol; LDL-C, low-density lipoprotein cholesterol; apoA-I, apolipoprotein A-I; ACEI, angiotensin-converting enzyme inhibitors; ARB, angiotensin II receptor blocker; CCB, calcium channel blocker.

by the STRING database (Fig. 4A and B). ApoA-I is likely functionally linked to the complement and coagulation cascades [with $\alpha$-2-macroglobulin (A2M)], renin-angiotensin system [with angiotensin-converting enzyme (ACE)] and the hypoxia-inducible factor (HIF)-1 signaling pathway (with
GAPDH). Using pathway analysis tools (http.//www.kegg.jp), several pathways were identified to be associated with apoA-I, including 'fat digestion and absorption', 'vitamin digestion and absorption' (with Cubilin) and the 'peroxisome proliferator activated receptor (PPAR) signaling pathway' (with fatty 


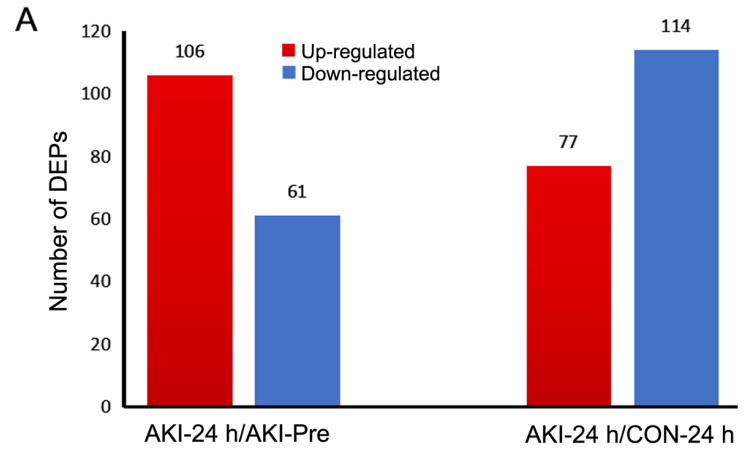

B

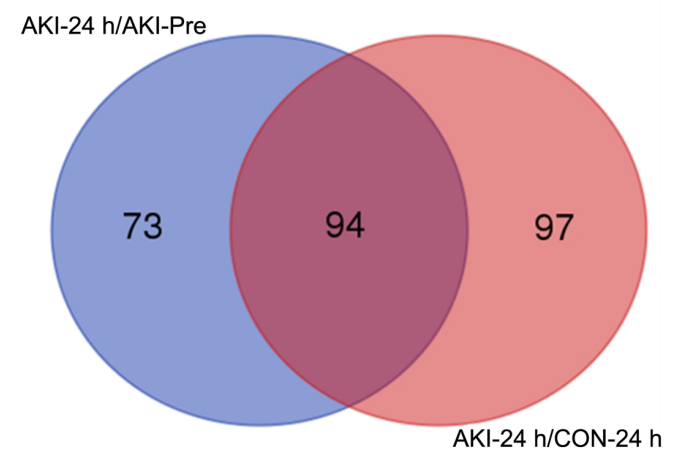

Figure 1. (A) DEPs identified in the comparisons of AKI-24 h/AKI-Pre (AKI group at $24 \mathrm{~h}$ post-PCI vs. pre-PCI) and AKI-24 h/CON-24 h (AKI group vs. CON group at $24 \mathrm{~h}$ post-PCI), (B) DEPs overlapped in both AKI-24 h/AKI-Pre and AKI-24 h/CON-24 h comparisons. AKI, acute kidney injury; CON, control; DEP, differentially expressed protein.

acid-binding protein 1). Relevant KEGG pathways are listed in Table SIV.

PRM validation results. In the validation set from 8 AKI- and $8 \mathrm{CON}$-group patients (AKI-v group vs. Control-v group; Table SVI), the mean age of AKI-v group was $75.50 \pm 9.63$ years vs. $74.00 \pm 4.95$ years of $\mathrm{CON}-\mathrm{v}$ group, and there were $62.5 \%$ males in AKI-v group vs. $75 \%$ in $\mathrm{CON}-\mathrm{v}$ group. The differential accumulation of the protein apoA-I was validated using PRM analyses. The fragments selected for quantification are presented in Table III. At $24 \mathrm{~h}$ after PCI, the protein expression levels of apoA-I displayed a 5.98-fold increase from the baseline levels, as well as a 2.09-fold increase compared with the CON group. The PRM results are consistent with the iTRAQ quantification results.

\section{Discussion}

At present, there is no single proven intervention or therapy for AKI. Using novel biomarkers for early detection of renal impairment, combined with preventive or supportive measures may improve patient outcomes (8). Urine is a useful sample for biomarker analysis, as it contains cellular elements, biochemicals and proteins which may reveal, at a given time-point, an individual's pathophysiological state. Proteomics approaches provide unbiased information on the protein changes in a biological sample as a whole, without pre-selection of potential targets of interest. In the last decade, tremendous progress has been made in urinary proteomics, in terms of uncovering novel biomarkers and potential therapeutic targets. In the present study, iTRAQ-based quantitative proteome analysis
Table II. Fold changes of apolipoprotein A-I expressed in AKI and control groups determined via isobaric tags for relative and absolute quantitation technology.

\begin{tabular}{lrr}
\hline Comparison & Fold change & P-value \\
\hline AKI-24 h/AKI-Pre & 18.8799 & 0.0338 \\
AKI-24 h/CON-24 h & 10.8643 & $<0.0001$ \\
CON-24 h/CON-Pre & 1.3305 & 0.0855 \\
AKI-Pre/CON-Pre & 0.0794 & 0.0695 \\
\hline
\end{tabular}

AKI, acute kidney injury; CON, control.

was performed for the identification of urinary biomarkers for PCI-AKI. iTRAQ has been demonstrated to be a useful tool for protein biomarker discovery. It markedly increases the identification sensitivity and quantitation accuracy of proteomic analyses and is more reliable than traditional two-dimensional electrophoresis (17). Using iTRAQ, the present study identified a significant rise in apoA-I levels in urine of patients with AKI as early as $24 \mathrm{~h}$ after PCI when compared to the baseline levels and to that of patients in the $\mathrm{CON}$ group, demonstrating that urinary apoA-I is a sensitive marker of renal injury after PCI in elderly patients. These results were then confirmed in an additional homogeneous cohort of individuals using the PRM-based targeted procedure, which has been demonstrated to have higher sensitivity and specificity for biomarker validation compared with the traditional antibody-based ELISA or western blotting approach (18).

These changed protein levels of apoA-I may also provide insight into the molecular mechanisms of action underlying the pathogenesis of PCI-AKI. While the pathophysiology of AKI following PCI has remained to be fully elucidated, two mechanisms of action, namely contrast media effects on renal perfusion and direct renal tubular toxicities, are thought to be mainly involved. The resultant reactive oxygen species-mediated oxidative stress brought about by these two mechanisms induces an inflammatory response in the kidneys, which may have an important role in the pathogenesis behind PCI-AKI $(5,19)$. HDL-cholesterol, in addition to its well-known reverse cholesterol transport effect, has anti-oxidative and anti-inflammatory properties (20). ApoA-I is the major apolipoprotein of HDL. Thiemermann et al (21) demonstrated that HDL alleviated renal dysfunction and injury in a rat model of renal ischemia/reperfusion (I/R). This capability of HDL was in part related to an independent property of apoA-I, which may reduce lipid peroxidation subsequent to oxidative stress in kidneys subjected to renal I/R. Administration of the synthetic apoA-I mimetic peptide $4 \mathrm{~F}$ resulted in the induction of nitric oxide synthase, preventing the release of proinflammatory cytokines and the restoration of renal function. Li et al (22) determined that apoA-I overexpression in mice had a protective effect on LPS-induced systemic inflammation and multiple organ injury including the kidneys through the suppression of endothelial cell adhesion molecules (intercellular adhesion molecule-1 and P-selectin), downregulation of NF- $\kappa \mathrm{B}$ and the inhibition of neutrophil activation. Hence, it is conceivable that raised expression levels of apoA-I in urine may suggest the 
A

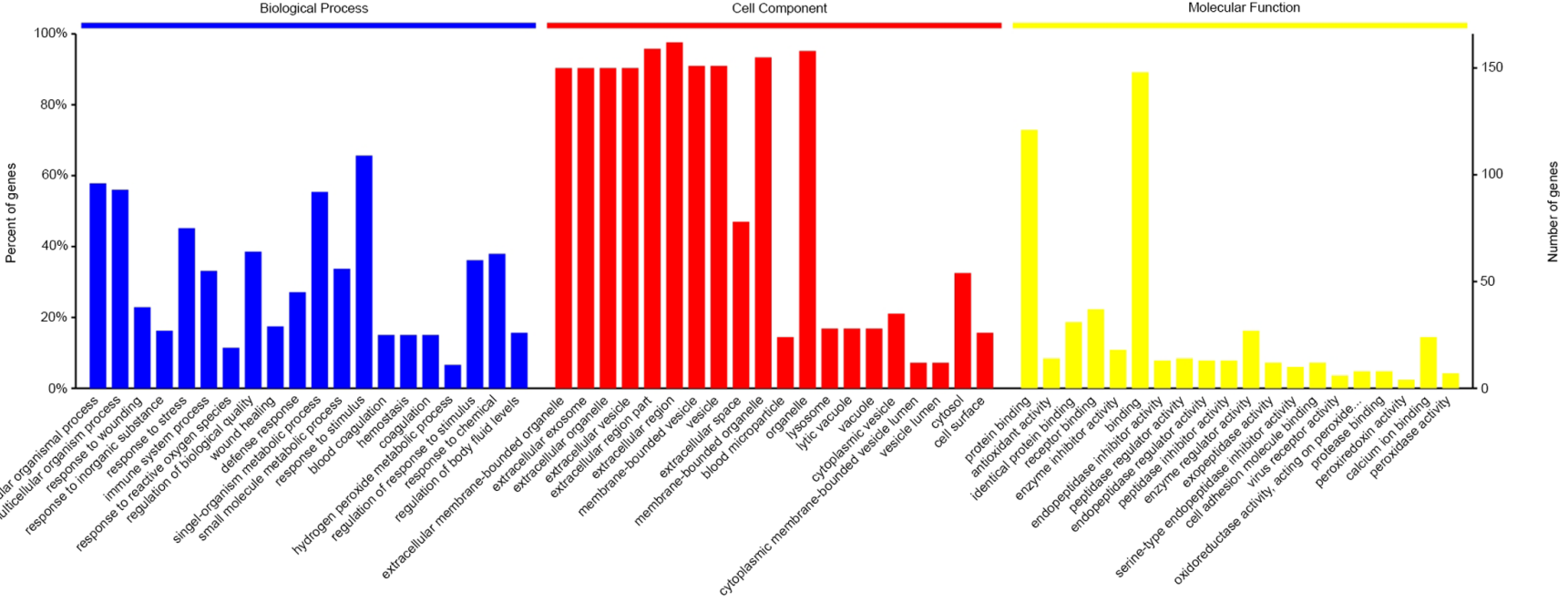

B

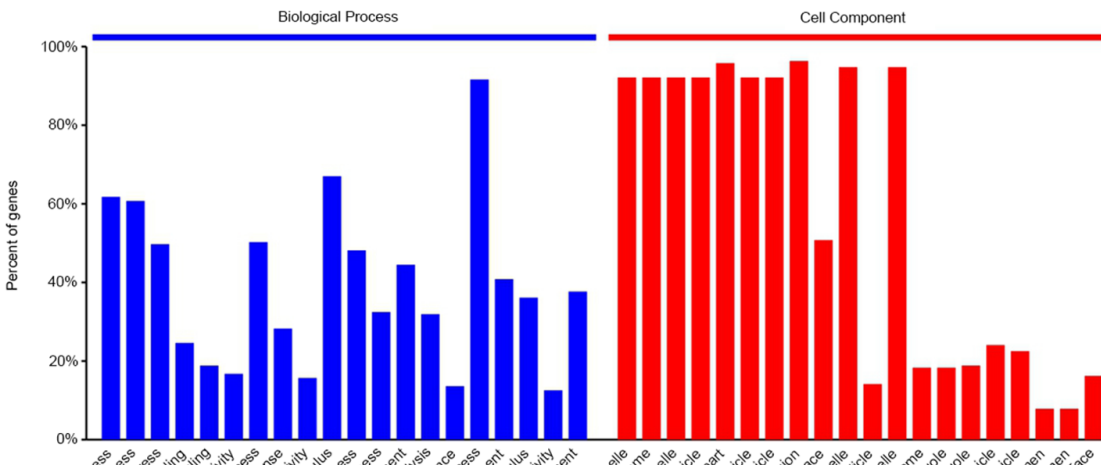

Molecular Function

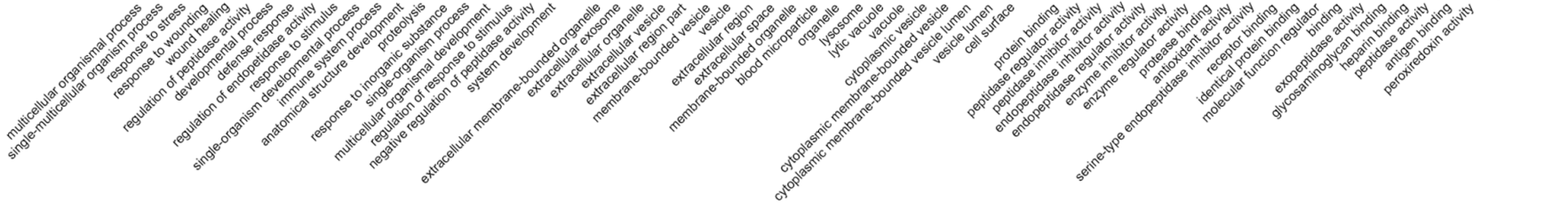

Figure 2. Gene ontology analysis of differentially expressed proteins between (A) AKI-24 h/AKI-Pre (AKI group at $24 \mathrm{~h}$ post-PCI vs. pre-PCI) and (B) AKI-24 h/CON-24 h (AKI group vs. CON group at $24 \mathrm{~h}$ post-PCI). The functional terms in the categories biological process, cellular component and molecular function are provided. AKI, acute kidney injury; CON, control.

absence of its protective role early in the course of PCI-AKI. Furthermore, the anti-oxidant and anti-inflammatory properties demonstrated in previous studies $(23,24)$, along with the bioinformatics analysis in the present study, revealed that the network interactions of proteins confirmed a close relationship between apoA-I and A2M, ACE, GAPDH and FABP1. These proteins may participate in the metabolism of apoA-I, which is associated with the complement and coagulation cascades, as well as the renin-angiotensin system, and the HIF-1 and the PPAR signaling pathways. These results indicated that the combination of these genes was responsible for the development of the AKI-group, which suggested potential directions for future research.

The data from the clinical tests in the present study also indicated that lower levels of plasma HDL-C and apoA-I prior to PCI were associated with a higher incidence of subsequent PCI-AKI. This result was not related to statin use or its dosage, consistent with previous studies. However, it is not clear whether low plasma HDL-C and apoA-I levels prior to PCI are related to the overexpression of urinary apoA-I after PCI, as the current experiment cannot distinguish whether urinary apoA-I was derived from plasma or the proximal tubules reflecting AKI. In addition, the present study enrolled patients with a wide range of clinical presentations. It was inevitable that patients with decreased renal function $(30<$ eGFR $<60 \mathrm{ml} / \mathrm{min}$ ) were involved in the present study. Recent studies have indicated that there is a relationship between patients with CKD and HDL-C dysfunction rather than HDL-C deficiency, leading to increased oxidative stress and inflammation (21). As such, further research may be performed to investigate the HDL-C/apoA-I quality during the development of PCI-AKI, particularly in high-risk populations.

The present study had several limitations. First, the study was a single-center study with a relatively small sample size. The patients enrolled were all older than 60 years with hypertension, CKD or diabetes. Although baseline-matched patients were selected as controls and pooled samples were analyzed to eliminate the individual heterogeneity, the nested case-control design of the present study may still have been predisposed to bias and residual confounding. However, this relatively non-selected patient population was used in the current study, which may reflect the real clinical condition. Further studies are required to replicate and validate the current findings. Moreover, due to the limited sample size of the present study, a multi-center study 

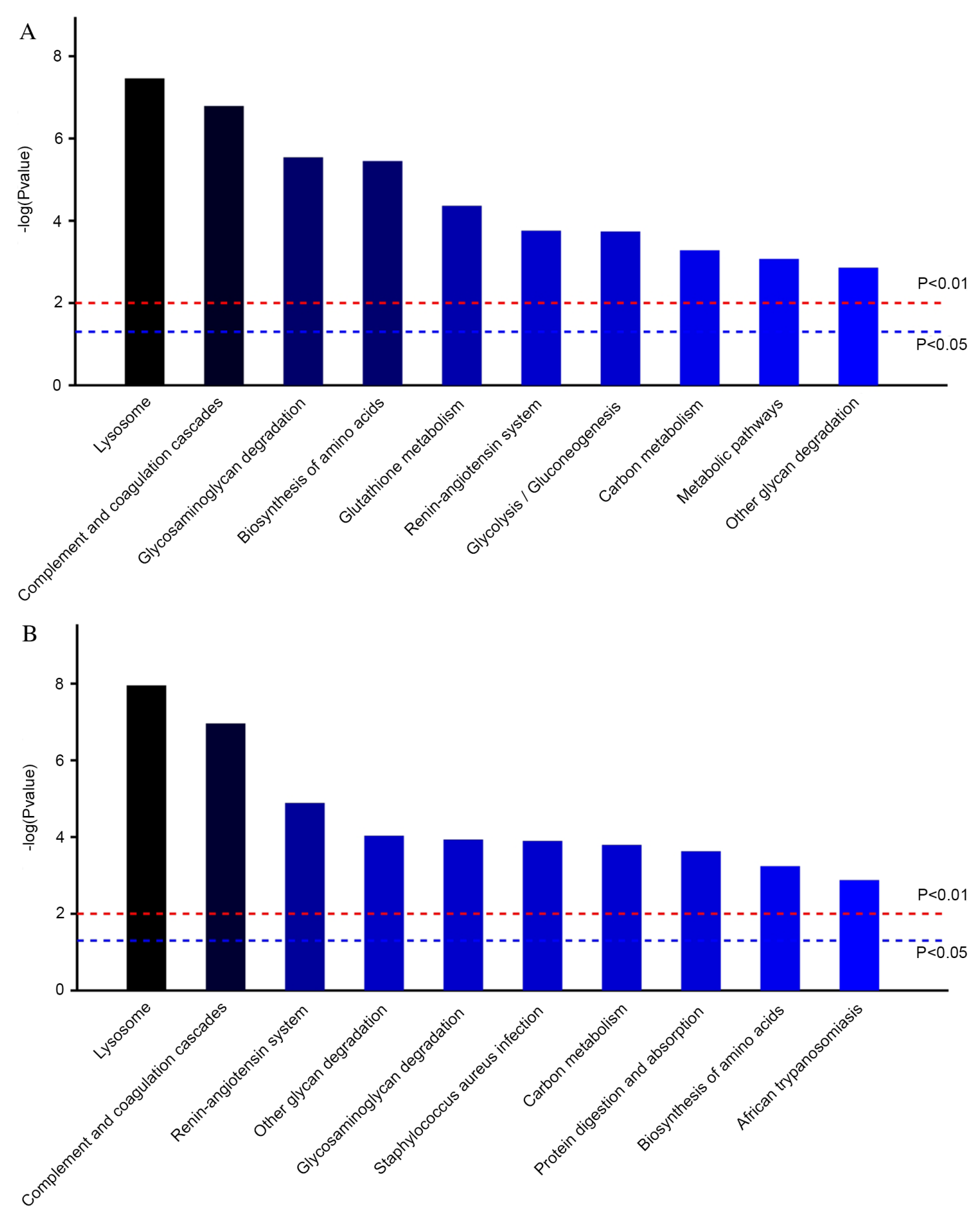

Figure 3. Kyoto Encyclopedia of Genes and Genomes pathways of differentially expressed proteins between (A) AKI-24 h/AKI-Pre (AKI group at $24 \mathrm{~h}$ post-PCI vs. pre-PCI) and (B) AKI-24 h/CON-24 h (AKI group vs. CON group at $24 \mathrm{~h}$ post-PCI). AKI, acute kidney injury; CON, control.

with a larger sample size would be needed. As another limitation, in the present study, apoA-I was the only protein selected for validation, which may have resulted in certain valuable proteins which are not related to lipids being neglected. There are likely to be other proteins, besides apoA-I, which may prove useful as biomarkers for PCI-AKI. Additional biomarker candidates from the current iTRAQ results should be further investigated in future studies to determine their clinical value in PCI-AKI. It is also possible that a multiple urine-protein panel consisting of multiple biomarkers may provide greater specificity and sensitivity than individual biomarkers alone. As such, a panel may be more valuable to be developed a reliable biomarker for PCI-AKI. Furthermore, all clinical information should be considered when evaluating biomarker data, including patients $<60$ years old, without hypertension, CKD or diabetes. Further analyses may be performed in the future, such as receiver operating characteristic or multivariate logistic regression, to better determine the marker ability of apoA-I for PCI-AKI.

In conclusion, apoA-I may be a promising biomarker candidate for early diagnosis ( $24 \mathrm{~h}$ post-contrast, prior to Scr levels rising) for PCI-AKI in elderly patients. It may be hypothesized that there are causal mechanisms involving apoA-I in PCI-AKI. In future studies, the role and regulation of apoA-I should be investigated further during the development of PCI-AKI, particularly the 
Table III. Peptides and protein abundance of apolipoprotein A-I expressed in AKI and control groups determined via parallel reaction monitoring technology.

\begin{tabular}{lccccc}
\hline Peptide & Charge & $\mathrm{m} / \mathrm{z}$ & AKI-Pre & AKI-24 $\mathrm{h}$ & CON-24 h \\
\hline EQLGPVTQEFWDNLEK & 2 & 966.9705 & $1.23 \times 10^{5}$ & $4.20 \times 10^{6}$ & $\mathrm{NA}$ \\
THLAPYSDELR & 2 & 651.3279 & $\mathrm{NA}$ & $6.09 \times 10^{6}$ & $2.87 \times 10^{5}$ \\
VQPYLDDFQK & 2 & 626.8141 & $5.22 \times 10^{6}$ & $8.74 \times 10^{6}$ & $1.72 \times 10^{6}$ \\
LLDNWDSVTSTFSK & 2 & 806.8963 & $\mathrm{NA}$ & $7.85 \times 10^{6}$ & $3.57 \times 10^{5}$ \\
ATEHLSTLSEK & 2 & 608.3144 & $2.64 \times 10^{6}$ & $9.05 \times 10^{5}$ & $\mathrm{NA}$ \\
General protein abundance & & & $2.66 \times 10^{6}$ & $5.56 \times 10^{6}$ & $9.30 \times 10^{5}$ \\
Fold change of apoA-I & & & & $5.98^{\mathrm{a}}$ & $2.09^{\mathrm{b}}$ \\
\hline
\end{tabular}

${ }_{\mathrm{a}}^{\mathrm{P}}<0.05, \mathrm{AKI}-24 \mathrm{~h}$ vs. AKI-Pre; ${ }^{\mathrm{b}}<0.05, \mathrm{AKI}-24 \mathrm{~h}$ vs. CON-24 h. AKI, acute kidney injury; CON, control; NA, not available; m/z, mass to charge ratio.
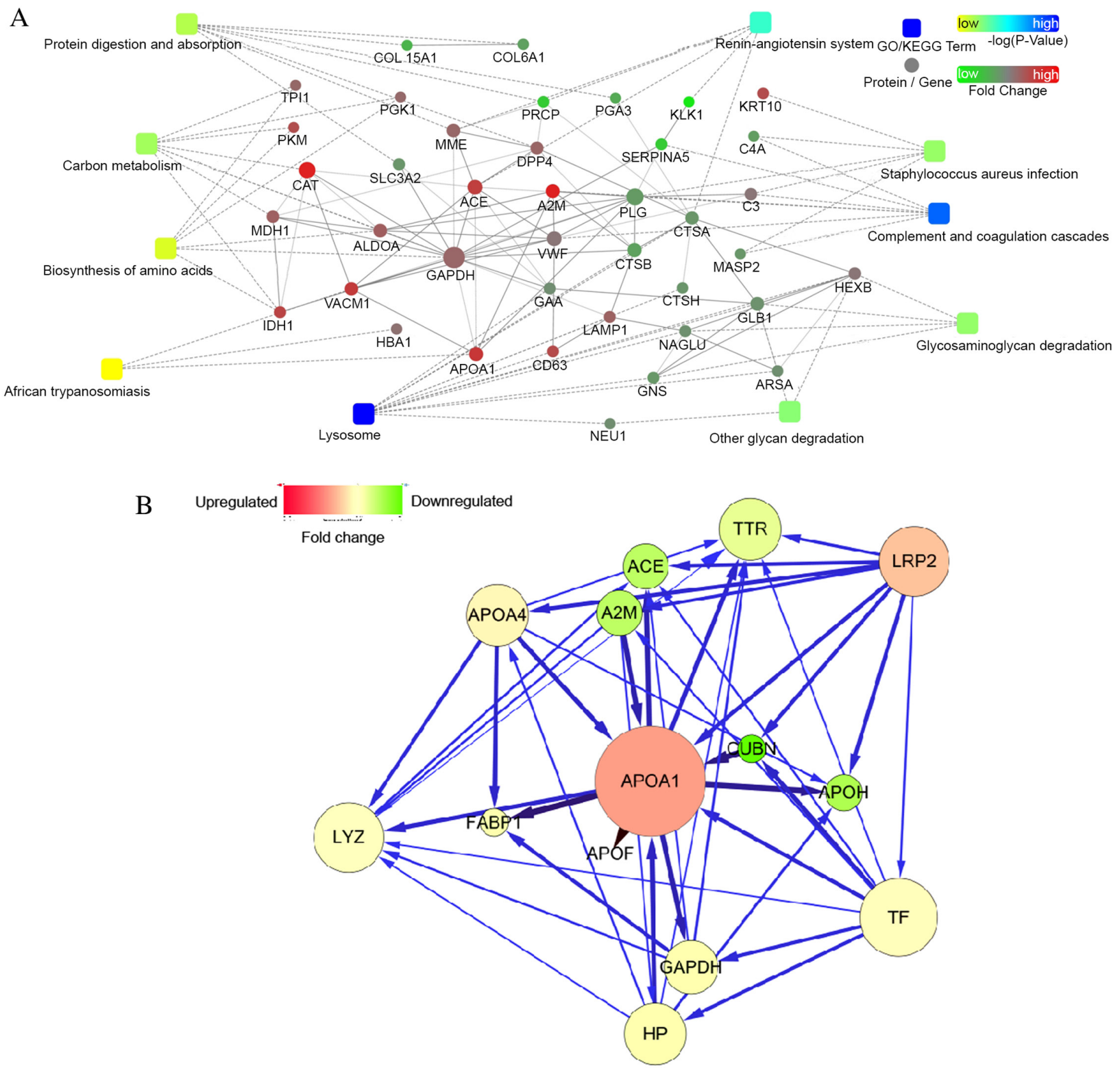

Figure 4. (A) Protein-protein interaction network of the differentially expressed proteins. (B) Protein-protein interaction network of apolipoprotein A-I. Upregulated proteins are indicated by red circles and downregulated proteins by dark green circles. Medium level of confidence (score 0.6282 , with the maximum level of confidence at 1 and the lowest at 0 ). A deeper shade of red/green indicates higher fold-changes. Thicker lines represent stronger interactions (according to scores of edge betweenness) and a bigger shape size indicates that the protein may interact with more proteins. GO, Gene Ontology; KEGG, Kyoto Encyclopedia of Genes and Genomes. 
relationship between plasma HDL-C and apoA-I levels prior to PCI and the raised expression levels of urinary apoA-I after PCI.

\section{Acknowledgements}

The authors would like to thank Dr Chenpin Shen (Department of Chemistry and Institutes of Biomedical Sciences, Fudan University, Shanghai, China) for helpful experimental assistance. Results presented in this study were included in a poster presentation (abstract no. SP242) at the 55th European Renal Association-European Dialysis and Transplant Association Congress in 2018 (Copenhagen, Denmark).

\section{Funding}

This study was supported in part by a project from the Medical Sciences Research Foundation of Zhejiang Province (grant no. 201336474 to QL) and Ningbo City Natural Sciences Research Foundation of Ningbo City (grant no. 2013A610266 to FZ). The funders had no role in the study design, data collection and analysis, decision to publish or preparation of the manuscript.

\section{Availability of data and materials}

The datasets used and/or analyzed during the present study are available from the corresponding author on reasonable request. The dataset generated in the present study was deposited at https://www.iprox.org (projectIDIPX0002961000; https://www. iprox.org//page/subproject.html?id=IPX0002961000).

\section{Authors' contributions}

FZ and QL conceived and designed the study. LHa, GS and LHu performed the experiments. HY contributed to acquisition of data. FZ contributed to the analysis and interpretation of data, and wrote the paper. QL and HY reviewed and edited the manuscript. All authors read and approved the final manuscript.

\section{Ethics approval and consent to participate}

The present study was approved by the ethics committee of HwaMei Hospital, University of Chinese Academy of Sciences (Ningbo, China). Written informed consent was obtained from each of the patients involved in the study.

\section{Patient consent for publication}

Not applicable.

\section{Competing interests}

The authors declare that they have no competing interests.

\section{References}

1. Ozkok S and Ozkok A: Contrast-induced acute kidney injury, A review of practical points. World J Nephrol 6: 86-99, 2017.

2. Chalikias G, Drosos I and Tziakas DN: Prevention of contrast-induced acute kidney injury: An update. Cardiovasc Drugs Ther 30: 215-228, 2016.

3. Fähling M, Seeliger E, Patzak A and Persson PB: Understanding and preventing contrast-induced acute kidney injury. Nat Rev Nephrol 13: 169-180, 2017.
4. McCullough PA, Choi JP, Feghali GA, Schussler JM, Stoler RM, Vallabahn RC and Mehta A: Contrast-induced acute kidney injury. J Am Coll Cardiol 68: 1465-1473, 2016.

5. Mamoulakis C, Tsarouhas K, Fragkiadoulaki I, Heretis I, Wilks MF, Spandidos DA, Tsitsimpikou C and Tsatsakis A: Contrast-induced nephropathy: Basic concepts, pathophysiological implications and prevention strategies. Pharmacol Ther 180: 99-112, 2017.

6. Andreucci M, Faga T, Riccio E, Sabbatini M, Pisani A and Michael A: The potential use of biomarkers in predicting contrast-induced acute kidney injury. Int J Nephrol Renovasc Dis 9: 205-221, 2016.

7. Luo Q, Zhou F, Dong H, Wu L, Chai L, Lan K and Wu M: Implication of combined urinary biomarkers in early diagnosis of acute kidney injury following percutaneous coronary intervention. Clin Nephrol 79: 85-92, 2013.

8. De Loor J, Gevaert K, Hoste E and Meyer E: How has urinary proteomics contributed to the discovery of early biomarkers of acute kidney injury? Expert Rev Proteomics 11: 415-424, 2014.

9. Sigdel TK, Gao Y, He J, Wang A, Nicora CD, Fillmore TL, Shi T, Webb-Robertson BJ, Smith RD, Qian WJ, et al: Mining the human urine proteome for monitoring renal transplant injury. Kidney Int 89: 1244-1252, 2016.

10. Luczak M, Formanowicz D, Marczak Ł, Suszyńska-Zajczyk J, Pawliczak E, Wanic-Kossowska $M$ and Stobiecki M: iTRAQ-based proteomic analysis of plasma reveals abnormalities in lipid metabolism proteins in chronic kidney disease-related atherosclerosis. Sci Rep 6: 32511, 2016.

11. Rao S, Walters KB, Wilson L, Chen B, Bolisetty S, Graves D, Barnes S, Agarwal A and Kabarowski JH: Early lipid changes in acute kidney injury using SWATH lipidomics coupled with MALDI tissue imaging. Am J Physiol Renal Physiol 310: F1136-F1147, 2016.

12. Park HS, Kim CJ, Hwang BH, Kim TH, Koh YS, Park HJ, Her SH, Jang SW, Park CS, Lee JM, et al: HDL cholesterol level is associated with contrast induced acute kidney injury in chronic kidney disease patients undergoing PCI. Sci Rep 6: 35774, 2016.

13. Smith LE, Smith DK, Blume JD, Linton MF and Billings FT IV: High-Density Lipoprotein cholesterol concentration and acute kidney injury after cardiac surgery. J Am Heart Assoc 6: e006975, 2017.

14. Shi T, Song E, Nie S, Rodland KD, Liu T, Qian WJ and Smith RD: Advances in targeted proteomics and applications to biomedical research. Proteomics 16: 2160-2182, 2016.

15. Kidney Disease: Improving Global Outcomes (KDIGO) Acute Kidney Injury Work Group: KDIGO clinical practice guideline for acute kidney injury. Kidney Int Suppl 2: 1-138, 2012.

16. Levey AS, Stevens LA, Schmid CH, Zhang YL, Castro AF III, Feldman HI, Kusek JW, Eggers P, Van Lente F, Greene T, et al: A new equation to estimate glomerular fltration rate. Ann Intern Med 150: 604-612, 2009.

17. Michael H, Justin D and Virginia E: Current state of the art for enhancing urine biomarker discovery. Expert Rev Proteomics 13: 609-626, 2016.

18. Chen Y, Mao P and Wang D: Quantitation of intact proteins in human plasma using top-down parallel reaction monitoring-MS. Anal Chem 90: 10650-10653, 2018.

19. Andreucci M, Faga T, Pisani A, Sabbatini M and Michael A: Acute kidney injury by radiographic contrast media: Pathogenesis and prevention. Biomed Res Int 2014: 362725, 2014.

20. Moreira RS, Irigoyen M, Sanches TR, Volpini RA, Camara NO, Malheiros DM, Shimizu MH, Seguro AC and Andrade L: Apolipoprotein A-I mimetic peptide $4 \mathrm{~F}$ attenuates kidney injury, heart injury, and endothelial dysfunction in sepsis. Am J Physiol Regul Integr Comp Physiol 307: R514-R524, 2014.

21. Thiemermann C, Patel NS, Kvale EO, Cockerill GW, Brown PA, Stewart KN, Cuzzocrea S, Britti D, Mota-Filipe H and Chatterjee PK: High density lipoprotein (HDL) reduces renal schemia/reperfusion injury. J Am Soc Nephrol 14: 1833-1843, 2003.

22. Li Y, Dong JB and Wu MP: Human ApoA-I overexpression diminishesLPS-induced systemic inflammation and multiple organdamage in mice. Eur J Pharmacol 590: 417-422, 2008.

23. van der Vorst EPC: High-density lipoproteins and apolipoprotein A1. Subcell Biochem 94: 399-420, 2020.

24. Tanaka S, Couret D, Tran-Dinh A, Duranteau J, Montravers P, Schwendeman A and Meilhac O: High-density lipoproteins during sepsis: From bench to bedside. Crit Care 24: 134, 2020.

This work is licensed under a Creative Commons

Attribution-NonCommercial-NoDerivatives 4.0 International (CC BY-NC-ND 4.0) License. 